\title{
NIELSEN NUMBERS OF MAPS OF TORI
}

ROBIN B. S. BROOKS, ROBERT F. BROWN, JINGYAL PAK AND DOUGLAS H. TAYLOR

ABSTRACT. The main result states that if $f: X \rightarrow X$ is any map on a $k$-dimensional torus $X$, then the Nielsen number and Lefschetz number of $f$ are related by the formula $N(f)=|L(f)|$. Thus, on the torus, the Lefschetz number gives information, not just on the existence of fixed points, but on the number of fixed points as well. No other compact Lie group has this property. The main result, when applied to certain types of maps on compact Lie groups, produces new information on the fixed point theory of such maps.

In the study of the fixed points of a map $f: X \rightarrow X$ on a connected finite polyhedron $X$, two numbers are associated with $f$; the Lefschetz number $L(f)$ and the Nielsen number $N(f)$. It is known that when $X$ is a circle, then $N(f)=|L(f)|$ for any map $f[3, \mathrm{p} .107]$. The purpose of this note is to prove that the same relationship holds for a map on any torus and to discuss consequences of this result.

The fixed point theory on which this paper is based can be found in [3] and $[5]$.

Theorem. Let $X$ be a k-dimensional torus and $f: X \rightarrow X$ any map. Then $N(f)=|L(f)|$.

Proof. The rational cohomology $H^{*}(X ; Q)$ is the exterior algebra on $k$ generators $\left\{x_{1}, \ldots, x_{k}\right\}$; where $x_{i} \in H^{1}(X ; Q)$. The map $f$ induces $f^{*}$ : $H^{*}(X ; Q) \rightarrow H^{*}(X ; Q)$. Let $f^{* j}: H^{j}(X ; Q) \rightarrow H^{j}(X ; Q)$ be the restriction of $f^{*}$ and set

$$
\begin{gathered}
f^{*}\left(x_{1}\right)=a_{11} x_{1}+\cdots+a_{1 k} x_{k} \\
\vdots \\
f^{*}\left(x_{k}\right)=a_{k 1} x_{1}+\cdots+a_{k k} x_{k}
\end{gathered}
$$

Let $M=\left[\alpha_{i j}\right]$ be the matrix associated in this way with $f^{* 1}$. The Lefschetz number $L(f)$ is given by

$$
\begin{aligned}
L(f) & =\operatorname{Tr}\left(f^{* 0}\right)-\operatorname{Tr}\left(f^{* 1}\right)+\cdots+(-1)^{k} \operatorname{Tr}\left(f^{* k}\right) \\
& =1-\left(\alpha_{11}+\cdots+\alpha_{k k}\right)+\cdots+(-1)^{k} \alpha_{11} \cdots \alpha_{k k} .
\end{aligned}
$$

Received by the editors May 3, 1974.

AMS (MOS) subject classifications (1970). Primary 55C20; Secondary 55F 10, 22E99.

Key words and phrases. Nielsen number, Lefschetz number, fixed point, compact Lie group, homogeneous space. 
But this is nothing else but

$$
\left|\begin{array}{ccc}
1-a_{11} & \cdots & a_{1 k} \\
a_{21} & \vdots \\
\vdots & \vdots \\
a_{k 1} & \cdots & \vdots \\
a_{k k}
\end{array}\right|=\operatorname{det}(I-M)
$$

so we conclude that $L(f)=\operatorname{det}(I-M)$. If $L(f)=0$ then $N(f)=0$ since $T(X)$ $=\pi_{1}(X)\left[3\right.$, p. 101]. Now assume $L(f) \neq 0$. Since $T(f)=\pi_{1}(X)$, then [7] states that $N(f)$ is the order of the cokernel of $1-f_{\#}$; where $f_{\#}: \pi_{1}(X) \rightarrow \pi_{1}(X)$ is induced by $f$. Now $\pi_{1}(X)=Z^{k}$, so we consider $1-f_{\sharp}: Z^{k} \rightarrow Z^{k}$. Since $X$ is a torus, we may assume $\pi_{1}(X)$ is generated by the duals $\left\{x_{1}^{\prime}, \ldots, x_{k}^{\prime}\right\}$ of $\left\{x_{1}, \ldots, x_{k}\right\}$. Represent $1-f_{\#}$ by an integer matrix $I-F$. There is a diagonal matrix $D=\operatorname{diag}\left(d_{1}, \ldots, d_{k}\right)$ such that $D=A(I-F) B$; where $A$ and $B$ are unimodular matrices. Now we have $\operatorname{det} D=\operatorname{det}(I-F)$ and the order of the cokernel of $1-f_{\#}$ is the order of the group $Z /\left(d_{1}\right) \oplus \cdots \oplus Z /\left(d_{k}\right)$. Thus the order of the cokemel of $1-f_{\#}$ is $\left|d_{1} \cdots d_{k}\right|=|\operatorname{det} D|=|\operatorname{det}(I-F)|$. But we have $\operatorname{det}(I-F)=\operatorname{det}(I-M)$ by duality and therefore $N(f)=|L(f)|$.

A generalization of this Theorem, for coincidences of maps between tori, can be found in [1, pp. 122-125].

Because of [3, p. 142], the Theorem implies that, given any map $f$ on a torus, there exists a map $g$ homotopic to $f$ such that $g$ has exactly $|L(f)|$ fixed points. In particular, if $L(f)=0$, i.e. if "one" is an eigenvalue of $f^{* 1}$, then there is a fixed point free map $g$ homotopic to $f$.

While it may be that the main result can be extended to some interesting class of spaces more general than tori, the following converse to the Theorem shows that such a class cannot include any other compact connected Lie group:

If $G$ is a compact connected Lie group such that $N(f)=|L(f)|$ for all maps $f: G \rightarrow G$, then $G$ is a torus.

To establish this observation, let $G$ be a compact connected nonabelian Lie group of rank $\lambda$, then $\pi_{1}(G) \cong Z^{r} \oplus F$ where $F$ is finite and $r<\lambda$. For $m \geq 2$, let $p_{m}: G \rightarrow G$ be defined by $p_{m}(g)=g^{m}$, then $N\left(p_{m}\right)$ is the order of the cokernel of the endomorphism of $\pi_{1}(G)$ which takes an element $\alpha$ to $(1-m) \alpha$. Therefore, $N\left(p_{m}\right)=(m-1)^{r}+\rho(m)$; where $\rho(m)$ is no larger than the order of $F$. On the other hand, $L\left(p_{m}\right)=(1-m)^{\lambda}[3$, p. 49], so $\lambda>r$ implies $N\left(p_{m}\right)<\left|L\left(p_{m}\right)\right|$ for $m$ sufficiently large.

Let $G$ be a compact connected Lie group, $H$ a connected subgroup, and $B=G / H$ the homogeneous space. Call a map $f: G \rightarrow G$ fibre-preserving if $f(x H) \subseteq f(x) H$ for all $x \in G$. Such a map induces a map $f^{\prime}: B \rightarrow B$. If $f$ has 
a fixed point, then $f\left(x_{0} H\right) \subseteq x_{0} H$ for some $x_{0}$; let $f_{0}$ be the restriction of $f$ to $x_{0} H$. By [7], $T(G)=\pi_{1}(G)$ and $T(B)=\pi_{1}(B)$ so all fixed point classes of $f$ have the same index, which we denote by $i(f)$, and all fixed point classes of $f^{\prime}$ have the same index $i\left(f^{\prime}\right)$.

Corollary, Let $G$ be a compact connected Lie group, $T^{k}$ a toral subgroup and $f: G \rightarrow G$ a fibre-preserving map such that $L(f) \neq 0$. Then

$$
i(f) N(f)= \pm i\left(f^{\prime}\right), v\left(f^{\prime}\right) \cdot v\left(f_{0}\right) \text {. }
$$

Proof Since the projection of $G$ onto $B=G / T^{k}$ is an orientable fibre bundle, $L(f)=L\left(f^{\prime}\right) L\left(f_{0}\right)[2]$, [6]. By [3, p. 99], $L(f)=i(f) N(f)$ and $L\left(f^{\prime}\right)=$ $i\left(f^{\prime}\right) N\left(f^{\prime}\right)$. The Theorem implies that $L\left(f_{0}\right)= \pm N\left(f_{0}\right)$.

The Corollary is still correct when $L(f)=0$, but it is then necessary to define $f_{0}$ more carefully (see [2]).

When $G=S^{3}$, the Corollary reduces to the counterexample of [4]. In fact, the Corollary may be viewed as the analogue to the Theorem of [4] for fibrepreserving maps on $G / T^{k}$.

\section{REFERENCES}

1. R. Brooks, Coincidences, roots and fixed points, Doctoral Dissertation, University of California, Los Angeles, Calif., 1967.

2. R. F. Brown, Fixed points and fibre maps, Pacific J. Math. 21 (1967), 465472. MR $35 \# 4921$.

3. - The Lefschetz fixed point theorem, Scott, Foresman, Glenview, Ill., 1971. MR $44 \# 1023$.

4. R. F. Brown and E. R. Fadell, Corrections to "The Nielsen number of a fibre map", Ann. of Math. (2) 95 (1972), 365-367. MR 47 \#5872.

5. E. Fadell, Recent results in the fixed point theory of continuous maps, Bull. Amer. Math. Soc. 76 (1970), 10-29. MR $42 \# 6816$.

6. - Review \#4921, Math. Rev. 35 (1968), 909.

7. Juang Bo-Ju (Chiang Po-chu), Estimation of the Nielsen numbers, Acta Math. Sinica 14 (1964), 304-312 = Chinese Math.-Acta 5 (1964), 330-339. MR $30 \# 1510$.

DEPARTMENT OF MATHEMATICS, B ATES COLLEGE, LEWISTON, MAINE 04240

DEPARTMENT OF MATHEMATICS, UNIVERSITY OF CALIFORNIA, LOS ANGELES, CALIFORNIA 90024

DEP ARTMENT OF MATHEMATICS, WAYNE STATE UNIVERSITY, DETROIT, MICHIGAN 48202 55101

DEP ARTMENT OF MATHEMATICS, H AMLINE UNIVERSITY, ST. P AUL, MINNESOTA 